\title{
Intra-pulse modulation recognition using short-time ramanujan Fourier transform spectrogram
}

\author{
Xiurong Ma*, Dan Liu and Yunlong Shan
}

\begin{abstract}
Intra-pulse modulation recognition under negative signal-to-noise ratio (SNR) environment is a research challenge. This article presents a robust algorithm for the recognition of 5 types of radar signals with large variation range in the signal parameters in low SNR using the combination of the Short-time Ramanujan Fourier transform (ST-RFT) and pseudo-Zernike moments invariant features. The ST-RFT provides the time-frequency distribution features for 5 modulations. The pseudo-Zernike moments provide invariance properties that are able to recognize different modulation schemes on different parameter variation conditions from the ST-RFT spectrograms. Simulation results demonstrate that the proposed algorithm achieves the probability of successful recognition (PSR) of over 90\% when SNR is above $-5 \mathrm{~dB}$ with large variation range in the signal parameters: carrier frequency (CF) for all considered signals, hop size (HS) for frequency shift keying (FSK) signals, and the time-bandwidth product for Linear Frequency Modulation (LFM) signals.
\end{abstract}

Keywords: Intra-pulse modulation recognition, Pseudo-Zernike moments, Short-Time Ramanujan Fourier Transform, Probability of successful recognition

\section{Introduction}

Intra-pulse modulation recognition aiming at recognizing the intentional intra-pulse modulation type of radar signals plays a critical role in modern intercept receivers, which could be used to recognize the signal threat level and choose the optimal algorithm to estimate parameters of the detected signal [1].

In intra-pulse modulation recognition context, more interest has been focused on the study of the feature based (FB) algorithms [2-5]. Thereinto, as a significant means to $\mathrm{FB}$, the time-frequency analysis has been developed because it allows description of the instantaneous characteristics of a signal in the two-dimensional (2D) timefrequency space [6-15]. The authors in [9] proposed a robust method for radar emitter recognition based on the Wigner-Ville distribution (WVD) and transfer learning, the average recognition rate (ARR) reaches more than

\footnotetext{
*Correspondence: lunwentoudi@163.com

The Department of Computer and Communication Engineering, Engineering Research Center of Communication Devices and Technology, Ministry of Education, Tianjin Key Laboratory of Film Electronic and Communication Devices, Tianjin University of Technology, 391, Binshui-West Road, 300384 Tianjin, Xiqing District, China
}

$90 \%$ when signal-to-noise ratio (SNR) is $10 \mathrm{~dB}$. In [10], Gustavo Lopez-Risueno et al. proposed an algorithm based on Short-time Fourier transform (STFT) to distinguish No Modulation, phase shift keying (PSK), frequency shift keying (FSK) and linear frequency modulation (LFM) sweeping a narrow band, it performs well when SNR is around $10 \mathrm{~dB}$. In [11], a morphological operation based method had been exploited for a recognition of constant hop size (HS), constant time-frequency product, and carrier frequency (CF) ranging from $500 \mathrm{MHz}$ to $1 \mathrm{GHz}$ intra-pulse modulations, the accuracy can reach more than 95\% for SNRs above $-4 \mathrm{~dB}$. In [12], Deguo Zeng et al. proposed an approach based on the ambiguity function to recognize six types of modulations, and it suitable for a recognition of LFM signals with bandwidth sweeping from $2 \mathrm{MHz}$ to $15 \mathrm{MHz}$, pulse-width (PW) equaling 3,5 and 7 $\mu s$ when SNRs above $-1 \mathrm{~dB}$. In [13], the authors utilized the Rihaczek distribution and the Hough transform (HT) to discriminate Monopulse (MP) and binary phase shift keying (BPSK) signals with limited CF, binary frequency shift keying (2FSK) and 4-ary frequency shift keying (4FSK) signals with limited HS, and LFM with large time- 
bandwidth product ranging from 17.5 to 65 , their simulation results show that the probability of successful recognition (PSR) is greater than $90 \%$ when the SNR is above $-4 \mathrm{~dB}$. However, these approaches suffer from low PSR under negative SNR environment, especially have certain limitations for recognizing radar signals with large variation range on CF, HS and time-bandwidth product in the complicated noise condition. Therefore, it is paramount to explore new robust algorithms to obtain high PSR under conditions of low SNR and to recognize signals in a large variation range of signal parameters.

Recently, the concept of Ramanujan Fourier Transform(RFT) based time-frequency transform, namely Short-time Ramanujan Fourier transform(ST-RFT) has been investigated owing to the good immunity to noise interference of RFT functions [16-18]. Following this, the time-frequency analysis of signals based on RFT was considered in a letter by Sugavaneswaran [19]. Their research indicates that in the presence of noise this class of transforms has lower effect in comparison to Discrete Fourier Transform (DFT) based time-frequency transforms. Consequently, regarding the noise robustness, the ST-RFT is more efficient than the traditional DFT based time-frequency transform, and is a promising solution for intra-pulse modulation recognition under low SNR.

Nonetheless, how to realize an efficient recognition procedure for radar signals with large parameter variation range is still a challenging problem. The pseudo-Zernike moments have opened a wider set of applications for radar signal recognition in recent years, because the moments can provide potentially useful invariance properties such as translation, scale, and rotational invariance [20,21]. In [22], Jarmo Lundén, et al. examined the suitability of pseudo-Zernike moments as features for radar waveform recognition, In [23], a new radar classification algorithm based on STFT and pseudo-Zernike moments features is proposed. Inspired by the aforesaid background, the pseudo-Zernike moments are beneficial to realize intrapulse modulation recognition in scenarios with large variation in the signal parameters.

The objective in this article is to develop a novel method which contains a "ST-RFT spectrogram computation", a "moments feature computation" and a "recognition" to realize a classification of MP, LFM, BPSK, 2FSK and 4FSK signals with large variation range in the signal parameters: $\mathrm{CF}, \mathrm{HS}$ and time-frequency product under negative SNR. The first part is used to obtain the ST-RFT spectrograms, which can represent features of intra-pulse modulation signals even when the SNR is low. In the second part, the pseudo-Zernike moments features are used to extract information on spectrograms, which can provide invariance properties that are able to recognize different modulations when parameters change. The last part is used to classify 5 modulations in detail. Simulation results have showed that the recognition algorithm achieves very reliable performance: over 90\% PSR when SNR is above $-5 \mathrm{~dB}$ with CF ranges from $800 \mathrm{MHz}$ to $1600 \mathrm{MHz}$, HS ranges from $60 \mathrm{MHz}$ to $1000 \mathrm{MHz}$, and the time-bandwidth product ranges from 8 to 500 .

The rest of the article is organized as follows. Section 2 proposes an intra-pulse modulation recognition model. Section 3 defines the mathematical model of the STRFT spectrogram and presents the spectrogram features for all the modulation schemes under consideration. Section 4 focuses on the mathematical model of pseudoZernike moments computation and describes the process of moments feature selection. Section 5 presents the proposed recognition algorithm. Simulation results are presented and discussed in Section 6. Finally, conclusions are presented in Section 7.

\section{System model}

An intra-pulse modulation recognition approach based on Short-Time Ramanujan Fourier Transform (ST-RFT) and pseudo-Zernike moments feature is proposed in this paper. The system model of the proposed approach is shown in Fig. 1.

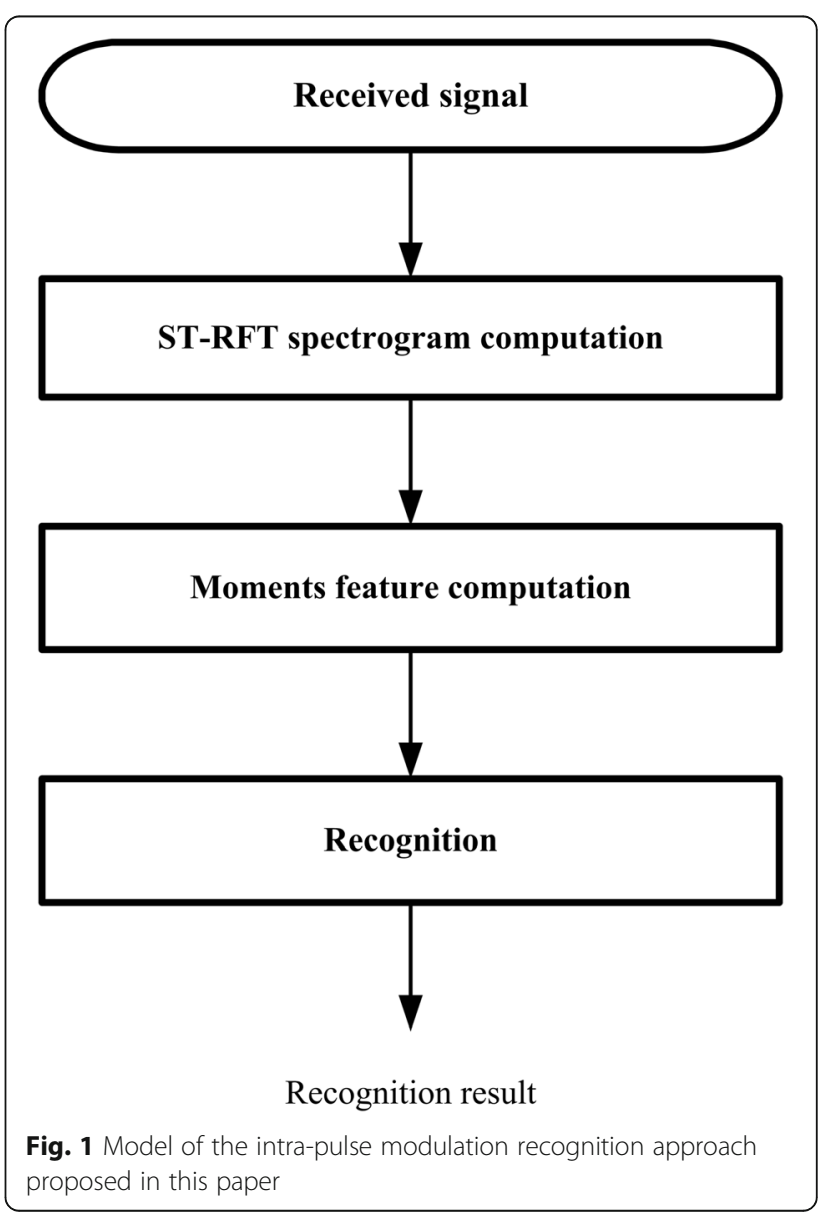


Three parts are included in this research: ST-RFT spectrogram computation, moments feature computation, and recognition. The ST-RFT analysis is a preprocess of moments feature computation, which could be used to obtain the ST-RFT spectrograms so as to represent features of intra-pulse modulation signals under negative SNR.

In the moments feature computation part, pseudoZernike moments features selected based on the degree of overlapping between each pair of classes of the signal data set are extracted from the spectrograms for its good invariance properties, which consist of $\psi_{3,3}$ feature and $\psi_{2,0}, \psi_{5,1}$ features.

After $\psi_{3,3}$ feature computation, intra-pulse modulation signals are described by vectors. These describing vectors are used for recognition by using threshold decision. Furthermore, after $\psi_{2,0}$ and $\psi_{5,1}$ features computation, intrapulse modulation signals are described by matrices, which are used for recognition by using KNN classifier [22].

\section{Mathematical model of ST-RFT spectrogram and ST-RFT spectrogram features}

3.1 Ramanujan Fourier transform (RFT)

In the classical DFT, the basis functions $e_{p}(n)$ are defined as [16]

$$
e_{p}(n)=\exp \left(2 j \pi \frac{p}{q} n\right), p=1, \ldots, q
$$

It is clear from $(1), e_{p}(n)$ are obtained as multiples of a basis frequency $(1 / q)$.

In the RFT, Ramanujan sums(RS) $c_{q}(n)$ are sums defined as the $n$-th powers of q-th primitive roots of unity [23]

$$
c_{q}(n)=\sum_{p=1 ;(p, q)=1}^{q} \exp \left(2 j \pi \frac{p}{q} n\right)
$$

It can be observed that $c_{q}(n)$ are the sums over the primitive characters $e_{p}(n)$. In other words, the basis functions are built by summing up components which are multiples of the same periodicity $q$, and only components satisfying $(p, q)=1$ contribute to the sum.

The sums were introduced by Ramanujan to play the role of base functions over which typical arithmetical functions $s(n)$ may be projected

$$
s(n)=\sum_{q=1}^{\infty} s_{q} c_{q}(n)
$$

It is obvious that an arithmetical function $s(n)$ is an infinite sequence defined for $1 \leq n \leq \infty$ for RFT, rather than that for DFT which is taken with a finite $n$ shown in [24].

The $s_{q}$ is referred to as the RFT coefficient given by [25]

$$
s_{q}=\frac{1}{\phi(q)} \lim _{N \rightarrow \infty} \frac{1}{N} \sum_{n=1}^{N} s(n) c_{q}(n)
$$

which is what we called the RFT.

Meanwhile, one can write the Wiener-Khintchine formula according to [26], and the linear property and the frequency multiplication property of RFT can be readily obtained.

\subsection{ST-RFT spectrogram computation}

In this paper, the ST-RFT is used to extract the necessary features of 5 modulations for intra-pulse modulation recognition. The reasons for this choice is that as a windowed RFT function, the ST-RFT transform allows simultaneous description of a signal in time and frequency so that the temporal evolution of the signal spectrum can be analyzed in the time-frequency space.

For an arbitrary discrete-time signal $s(n)$ of length $\mathrm{N}$, the ST-RFT of the signal is defined as

$$
S T-R F T_{s}(k, q)=\frac{1}{\phi(q)} \lim _{N \rightarrow \infty} \frac{1}{N} \sum_{n=1}^{N} s(n) \varphi^{*}(n-k) c_{q}(n),
$$

where $\phi(k)$ is the Rectangular window function of length $\mathrm{H}$, and $\phi(0)=1$.

Then the ST-RFT spectrogram $S_{s}(k, q)$ defined as the squared absolute value of the ST-RFT of $s(n)$ is given by

$$
S_{s}(k, q)=\left|S T-R F T_{s}(k, q)\right|^{2} .
$$

In the present work here, we take MP signal as an example to illustrate the deduction of the ST-RFT spectrogram expression of 5 modulations: MP signal, LFM signal, BPSK signal, 2FSKsignal and 4FSK signal.

Considering the following continuous-time MP signal

$$
s_{M P}(t)=A e^{j\left(2 \pi \frac{1}{T} t+\varphi_{0}\right)},
$$

where $f_{c}$ is the carrier frequency(CF), $T=\frac{1}{f_{c}}$ is the period of the continuous-time signal, $A$ and $\phi_{0}$ are the amplitude and the initial phase of MP separately.

For a sampling interval of $T_{s}$ (the sampling frequency (SF) is $f_{s}=\frac{1}{T_{s}}$ ), the discrete representation of signal (7) then becomes

$$
s_{M P}\left(n T_{s}\right)=A e^{j\left(2 \pi n \frac{T_{s}}{T}+\varphi_{0}\right)} .
$$

Let us represent $\frac{T_{s}}{T}$ as $\frac{1}{T_{0}}$, the expression of (8) can be given as

$$
s_{M P}(n)=A e^{j\left(2 \pi n \frac{1}{T_{0}}+\varphi_{0}\right)},
$$

where $T_{0}$ represents the number of samples in one cycle can be written as $T_{0}=\frac{f_{s}}{f_{c}}$. 
Using Eq. (5), the ST-RFT of MP can be expressed as

$$
S T-\operatorname{RFT}_{M P}\left(k, T_{0}\right)=\frac{A e^{j\left(2 \pi \frac{(-k+1)}{T_{0}}+\phi_{0}\right)}}{\phi\left(T_{0}\right)},
$$

in the case of $q=T_{0}$.

Substituting Eq. (10) into Eq. (6), the ST-RFT spectrogram of MP becomes

$$
S_{M P}\left(k, T_{0}\right)=\left|S T-R F T_{M P}\left(k, T_{0}\right)\right|^{2}=\frac{A^{2}}{\left(\phi\left(T_{0}\right)\right)^{2}} .
$$

\subsection{ST-RFT spectrogram features}

\subsubsection{Analysis of ST-RFT spectrogram features}

In practice, there exists a tradeoff between time and frequency resolution when determining the window length (the duration of window), that is to say, a long duration of window will provide a poor frequency resolution and vice versa. Through a series of simulation experiments, a Rectangular window of length $H=\frac{4000}{10}=400$ is selected, which can provide the best frequency resolution-time resolution tradeoff for 5 modulations above-mentioned. Examples of amplitude normalized ST-RFT spectrograms $P_{s}(k, q)$ (a normalization with respect to its maximum value of each ST-RFT spectrogram $S_{s}(k, q)$ )of 5 modulations computed from a sample of length $N=4000$ with a Rectangular window of length $H=400$ are shown in Fig. 2a-e. The contours on the plot represent relative magnitude with the horizontal axis as $\mathrm{q}$ and the vertical axis as $k(\mu s)$.

Figure 2 shows the amplitude normalized ST-RFT spectrograms $P_{s}(k, q)$ reflecting time-frequency distribution features of 5 types of modulation signals. Fig.2a shows the $P_{M P}(k, q)$ for MP signal. Ideally, there would be a straight line centred about $T_{0}$ in $k-q$ plane as the Eq. (11) implied. By contrast, Fig. 2a shows the line to be spread out in $q$ direction at the expense of reduced frequency resolution, and the peak energy is mainly concentrated in the location of $T_{0}$. The $P_{L F M}(k, q)$ for LFM signal with chirp rate $u=300$ as depicted in Fig.2b. Based on the observation of the spectrogram, the spectrum line can be approximated by a piecewise line starting at $T_{0}$ and finishing at $T_{0}-i$,where $i=1,2, \ldots T_{0}$ -1 , and each segment reflects the change of its frequency and phase. The $P_{B P S K}(k, q)$ for BPSK signal shown in Fig. 2c illustrates that the amplitude of spectrum obtains the minimum at instant of time of phase conversion, and in the duration of intercode, the $P_{B P S K}(k, q)$ is the same as the $P_{M P}(k, q)$. The $P_{2 F S K}(k, q)$ for 2FSK signal can be seen in Fig. 2d, which has five vertical line segments centered about $T_{0}$ and $T_{1}$ in $k-q$

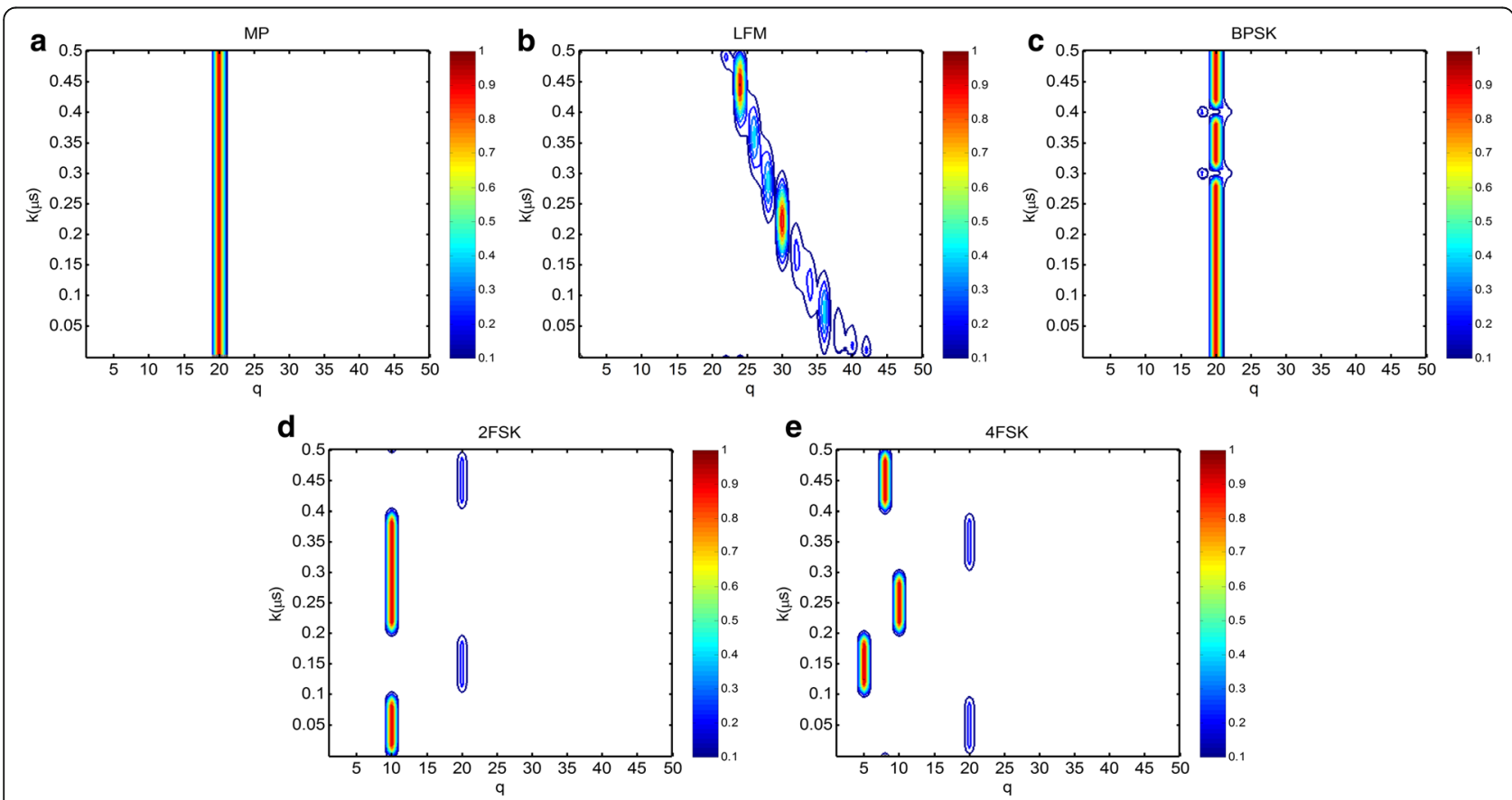

Fig. 2 Examples of amplitude normalized ST-RFT spectrograms $P_{5}(k, q)$ computed from a sample of length $N=4000$ with a Rectangular window of length $H=400$ for 5 modulations: (a) $P_{M P}(k, q)$ for MP signal; (b) $P_{L F M}(k, q)$ for LFM signal; (c) $P_{B P S K}(k, q)$ for BPSK signal encoded by Barker codes; (d) $P_{\text {2FSK }}(k, q)$ for 2FSK signal encoded by deterministic codes $\left[\begin{array}{lllll}1 & 0 & 1 & 1 & 0\end{array}\right]$; (e) $P_{4 F S K}(k, q)$ for 4FSK signal encoded by deterministic codes $\left[\begin{array}{lllll}0 & 3 & 1 & 0 & 2\end{array}\right]$ 
plane embodying the number of frequency points, while for the $P_{4 F S K}(k, q)$ of 4 FSK signal has 5 ones centered about $T_{0}, T_{2}, T_{1}, T_{0}, T_{3}$ in k-q plane as shown in Fig. $2 \mathrm{e}$.

In summary, the contours on the plot show different spectrogram features of 5 modulations. Hence, the STRFT spectrograms can serve as a discriminating feature.

\subsubsection{Analysis of discriminability}

The parameter $R$ giving the similarity degree between two amplitude normalized spectrogram $P_{s 1}(k, q)$ and $P_{s 2}(k, q)$ is defined as

$$
R=\frac{\sum_{k=1}^{N} \sum_{q=1}^{N}\left(P_{s 1}(k, q) \times P_{s 2}(k, q)\right)}{\sqrt{\sum_{k=1}^{N} \sum_{q=1}^{N}\left(P_{s 1}(k, q)\right)^{2}} \sqrt{\sum_{k=1}^{N} \sum_{q=1}^{N}\left(P_{s 2}(k, q)\right)^{2}}} .
$$

The similarity degree is bounded, $0 \leq R \leq 1$.

The similarity between any two amplitude normalized ST-RFT spectrograms computed by Eq. (12) with respect to different modulations in the absence of noise is depicted in Table 1. Intuitively, the MP and BPSK signals are difficult to distinguish from each other due to the fact that the spectrograms of the two modulations are similar enough with a similarity degree of 0.9341 . In addition, for MP and LFM signals as well as LFM and BPSK signals, the corresponding $R$ are 0.4312 and 0.4544 respectively that means this feature is considered not reliable to provide an effective method of signal differentiation.

Furthermore, the theoretical analysis in Section 3.2 indicates that the location of the ST-RFT spectral peak will be shifted induced by the variation of CFs of the input signals, then alter the feature value $P_{s}(k, q)$ and will finally influence the recognition results.

To tackle these problems, we propose a novel signal recognition method that is based on the combination of the ST-RFT spectrogram and the pseudo-Zernike moments.

Table 1 Similarity between two $P_{s}(k, q)$ with respect to different modulations in the absence of noise

\begin{tabular}{llllll}
\hline Type & MP & LFM & BPSK & 2FSK & 4FSK \\
\hline MP & 1 & 0.4312 & 0.9341 & 0.1241 & 0.1286 \\
LFM & & 1 & 0.4544 & 0.0480 & 0.0750 \\
BPSK & & & 1 & 0.1248 & 0.1291 \\
2FSK & & & & 1 & 0.3258 \\
4FSK & & & & & 1 \\
\hline
\end{tabular}

\section{Mathematical model of pseudo-Zernike} moments and moments feature selection

\subsection{Mathematical model of pseudo-Zernike moments}

Moments have been widely used in image processing for pattern recognition due to its useful invariance properties such as translation, scale, and rotational invariance [21, 27]. Such features capture global information about the image and do not require closed boundaries as boundarybased methods such as Fourier descriptors [27].

The formation of polar coordinates of the pseudoZernike moments for $f(x, y)$ can be obtained by projecting $f(x, y)$ onto orthogonal pseudo-Zernike polynomials $R_{e, m}(\rho) e^{i e \theta}$, by the integral [28].

$$
\psi_{e, m}=\frac{e+1}{\pi} \int_{0}^{2 \pi} \int_{0}^{1} R_{e, m}(\rho) e^{-i m \theta} f(\rho \cos \theta, \rho \sin \theta) \rho d \rho d \theta,
$$

where $\rho=\sqrt{x^{2}+y^{2}}$ represents the distance from the origin to a point in the $x-y$ plane, and $\theta=\arctan \frac{y}{x}$ is a counterclockwise angular displacement in radians from the positive $x$-axis. $R_{e, m}(\rho)$ are the radial polynomials expressed as

$$
R_{e, m}(\rho)=\sum_{l=0}^{e-|m|}(-1)^{l} \frac{(2 e+1-l) !}{l !(e+|m|+1-l) !(e-|m|-l) !} \rho^{e-l},
$$

where $e=0,1,2, \ldots ., \infty$ is the degree of the polynomial, $\mathrm{m}$ represents its angular dependence, which takes on positive and negative integer values subject to $e \geq|m|$ only.

\subsection{The translation invariance of pseudo-Zernike moments}

The translation invariance [27] of the pseudo-Zernike moments is suitable to be applied in illustrating effects of the variation of CFs and is utilized as time-frequency spectrogram features in radar signal classification.

For the amplitude normalized spectrogram $P_{s}(k, q)$ of the 5 modulations, the translation invariance is done by transforming the original time-frequency spectrogram $P_{s}(k, q)$ into another one which is $P_{s}(k+\bar{k}, q+\bar{q})$, where $\bar{k}$ and $\bar{q}$ are the centroid location of $P_{s}(k, q)$ computed from

$$
\bar{k}=\frac{m_{10}}{m_{00}}, \bar{q}=\frac{m_{01}}{m_{00}},
$$

where $m_{00}$ is the zero order moment defined as $m_{00}$ $=\sum_{k} \sum_{q} P_{s}(k, q), m_{01}$ and $m_{10}$ are first order moments, given by $m_{10}=\sum_{k} \sum_{q} k P_{s}(k, q)$ and $m_{01}=$ $\sum_{k} \sum_{q} q P_{s}(k, q)$.

In other words, the origin is moved to centroid before moment comoutation. 
In the present work here, a parameter $\delta$ is presented to illustrate the translation invariance of the pseudoZernike moments is suitable to be applied in signal recognition when CFs change.

$$
\delta=\left.\left|\psi_{e_{i}, m_{i}}\right|\right|_{C F \neq 1600 M H z}-\left.\left|\psi_{e_{i}, m_{i}}\right|\right|_{C F=1600 M H z}, i=1,2,
$$

where $\psi_{e_{1}, m_{1}}=\psi_{2,0}, \psi_{e_{2}, m_{2}}=\psi_{4,2}$

As Fig. 3 shows, the maximum variations of $\delta$ in $\left|\psi_{2,0}\right|$ for MP and LFM signals are $2.31 \times 10^{-6}$ and $2 \times 10^{-6}$, and the maximum variations of $\delta$ in $\left|\psi_{4,2}\right|$ for MP and LFM signals are $3.5 \times 10^{-6}$ and $3.48 \times 10^{-6}$, all the values are very small. Consequently, the features are nearly invariant to $\mathrm{CFs}$ changing and are feasible for signal classification with the variation in the signal CFs.

\subsection{Pseudo-Zernike moments feature selection}

The overlap measure indicates the degree of overlapping between two clusters, which can be quantified by computing an inter-cluster overlap [29]. A definition of the overlap rate(OLR) was proposed in [30], which is utilized as representative of the degree of overlap between the given two clusters $C_{i}$ and $C_{j}$. The OLR is determined by the ratio of the number of the overlap points to that the number of small cluster's points.

$$
\operatorname{OLR}\left(C_{i}, C_{j}\right)= \begin{cases}1, & \text { if } N_{\text {Over-Region }} \geq N_{\min }, \\ \frac{N_{\text {Over }_{\text {region }},}}{N_{\min }} & \text { others. }\end{cases}
$$

where $N_{\text {Over_Region }}$ represents the number of the overlap points, $N_{\min }$ is the minimum value of $N_{i}$ and $N_{j}$, which stands for the number of points in each cluster

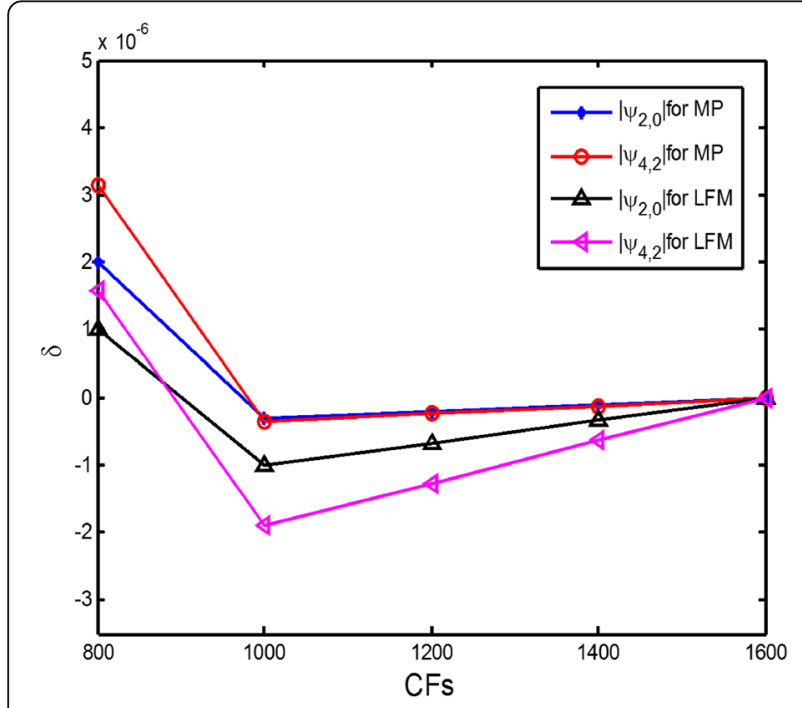

Fig. 3 The values of $\delta$ versus different CFs of MP and LFM with $u=80$ separately. The $\operatorname{OLR}\left(C_{i}, C_{j}\right)$ varies from 0 to1, the closer the $\operatorname{OLR}\left(C_{i}, C_{j}\right)$ is to 0 , the better the cluster separation is. Conversely, the closer the $\operatorname{OLR}\left(C_{i}, C_{j}\right)$ is to 1 , the two clusters become more strongly overlapped.

In the following, three pseudo-Zernike moment features based on the average value of $\operatorname{OLR}\left(O L R^{\prime}\right)$ are proposed for signal recognition. Here the signal data projected onto the 2-D/4-D feature space is obtained by testing all features of the pseudo-Zernike moments ranging from order 1 to order 6 .

The algorithm of the moments feature selection for distinguishing LFM with the time-bandwidth between 8 and 500 in the case of SNR varying from $-5 \mathrm{~dB}$ to $5 \mathrm{~dB}$ from the rest of signals is summarized as follows:

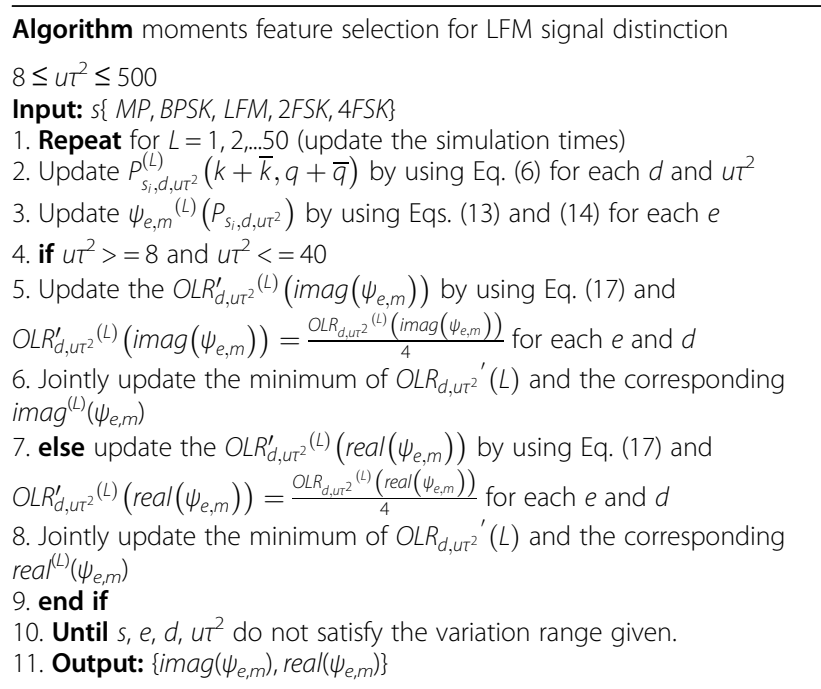

step 4: Combining the advantages of $\operatorname{imag}\left(\psi_{3,3}\right)$ and $\operatorname{real}\left(\psi_{3,3}\right)$ to discriminate LFM signals with large variation range in the time-bandwidth product from other modulations.

The $\psi_{3,3}$ is computed as

$$
\begin{aligned}
& \psi_{3,3}=\frac{4}{\pi} \int_{0}^{2 \pi} \int_{0}^{1} R_{3,3}(\rho) e^{-i 3 \theta} P_{\tilde{s}}(\rho \cos \theta, \rho \sin \theta) \rho d \rho d \theta \\
& R_{3,3}(\rho)=\rho^{3} .
\end{aligned}
$$

For other signals classification, we tested all combinations of two features ranging from order 1 to order 6 and measured the $O L R^{\prime}=\frac{\sum O L R}{6}$ which is defined as the average value of OLR between different classes taken in the 4-D feature space and find the minimum. Following the foresaid algorithm, the 8th order moments of index 5 versus index 27 for pseudo-Zernike moments 
referring to $\psi_{2,0}$ and $\psi_{5,1}$ are experimentally selected as features, which specifically suitable for signal classification with the exception of LFM.

The $\psi_{2,0}$ and $\psi_{5,1}$ are given by

$$
\begin{aligned}
& \psi_{2,0}=\frac{3}{\pi} \int_{0}^{2 \pi} \int_{0}^{1} R_{2,0}(\rho) P_{\tilde{s}}(\rho \cos \theta, \rho \sin \theta) \rho d \rho d \theta \\
& \psi_{5,1}=\frac{6}{\pi} \int_{0}^{2 \pi} \int_{0}^{1} R_{5,1}(\rho) e^{-i \theta} P_{\tilde{s}}(\rho \cos \theta, \rho \sin \theta) \rho d \rho d \theta
\end{aligned}
$$

$$
\begin{aligned}
R_{2,0}(\rho) & =10 \rho^{2}-12 \rho+2, R_{5,1}(\rho)=330 \rho^{5}-840 \rho^{4} \\
& +756 \rho^{3}-280 \rho^{2}+35 \rho .
\end{aligned}
$$

\section{Recognition algorithm}

\subsection{Steps of the proposed algorithm}

The proposed modulation signal recognition algorithm is shown in Fig. 4.

The starting point are the modulation signals $\tilde{s}(n), n$ $=0,1, \ldots, N-1$ to which Gaussian white noise is added. And the following steps of classifying various modulation types of signals are shown as follows:

Step 1: ST-RFT spectrogram Computation.

Step 1.1 Computing the amplitude normalized ST-RFT spectrogram $P_{\tilde{s}}(k, q)$ of 5 modulations mentioned-above.
Step 1.2 Computing the centroid moved amplitude normalized ST-RFT spectrogram $P_{\tilde{s}}(k+\bar{k}, q+\bar{q})$.

Step 2: LFM signal classification.

Measuring $\operatorname{imag}\left(\psi_{3,3}\right)$ and $\operatorname{real}\left(\psi_{3,3}\right)$ respectively. If $\operatorname{imag}\left(\psi_{3,3}\right)>t h_{L F M_{-} 1}$ or $\operatorname{real}\left(\psi_{3,3}\right)<t h_{L F M_{2}}$, the signal is regarded as LFM, else go to step 3.

Step 3: Other signals classification.

Step 3.1 Pseudo-Zernike moments $\psi_{2,0}$ and $\psi_{5,1}$ computation.

Step 3.2 Constructing the 2-D feature space by using $\psi_{2,0}$ and $\psi_{5,1}$ and determining the optimal distribution range of the spectrogram features of different modulations from the feature space.

Step 4: Use a K-nearest neighbour(KNN)classifier to assign each element to a class for the input radar signals, to perform the classification procedure.

\subsection{The thresholds for LFM signals recognition}

The thresholds $t h_{L F M_{-} 1}$ and $t h_{L F M_{-} 2}$ are utilized to distinguish LFM with the time-bandwidth product between 8 and 40 and to distinguish LFM with the timebandwidth product between 41 and 500 from other signals. They could be obtained by the iterative thresholding algorithm [20] and lots of simulations.

As in Fig. 5a, the minimum of the average value of $\operatorname{imag}\left(\psi_{3,3}\right)$ for LFM signal class is obtained when $u \tau^{2}=$ 40 at $S N R=-5 d B$ which is close to $0.40 \times 10^{-4}$ and for the rest of other signal classes the maximum of the average value of $\operatorname{imag}\left(\psi_{3,3}\right)$ obtained at $S N R=5 d B$ is close to

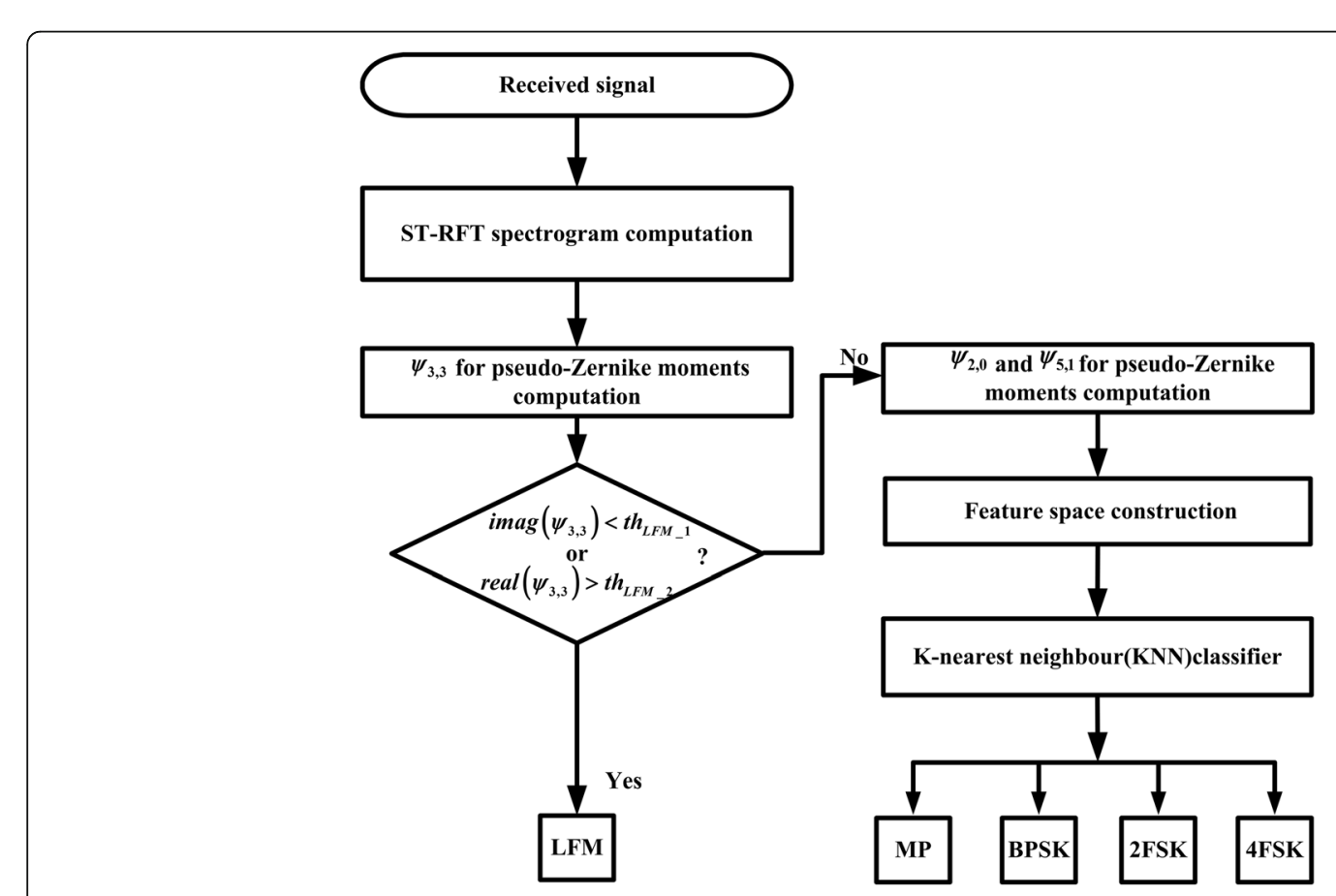

Fig. 4 Block scheme of proposed recognition algorithm 

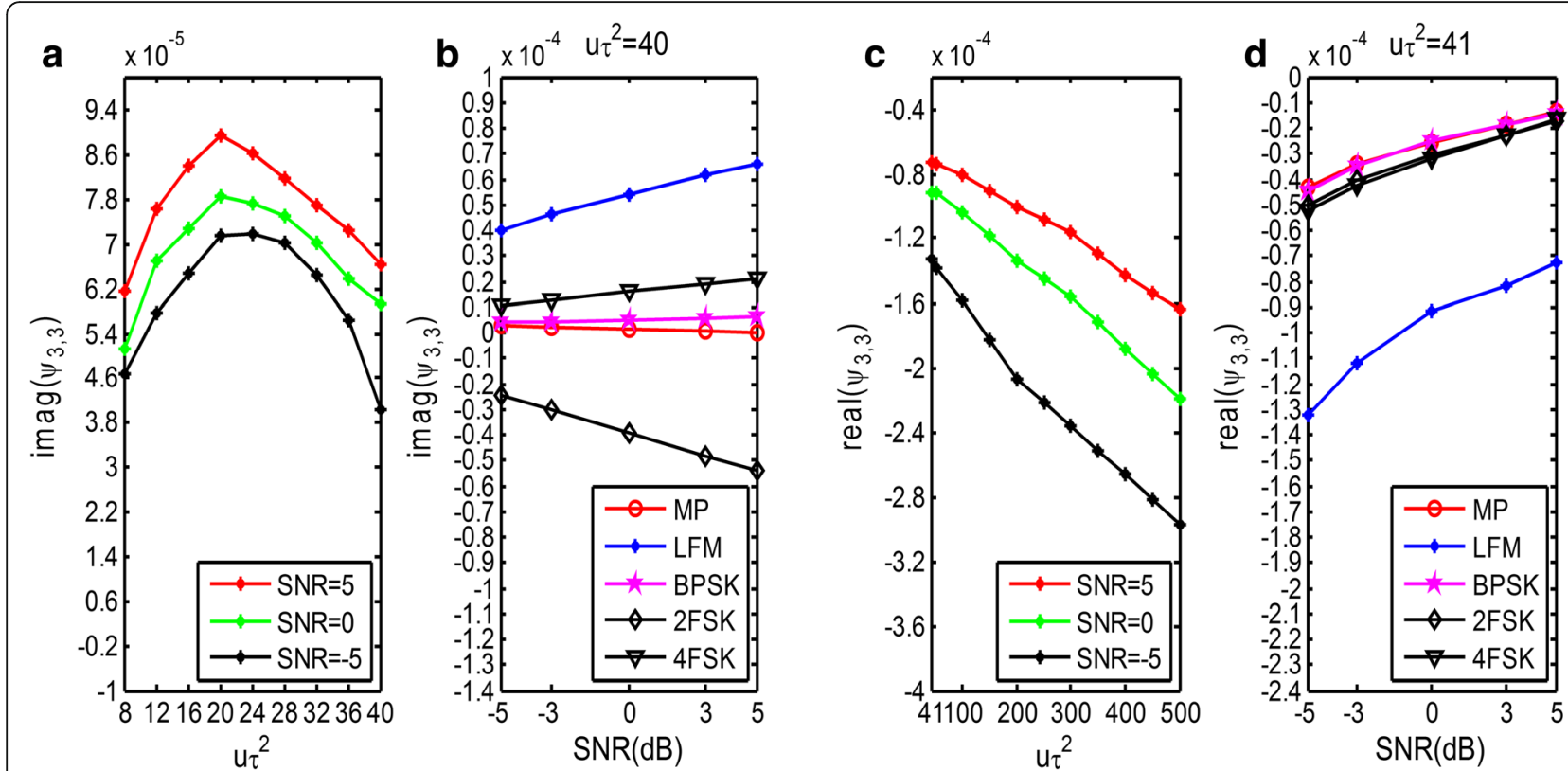

Fig. 5 The thresholds for LFM signals recognition: a the average values of $\operatorname{imag}\left(\psi_{3,3}\right)$ against different $u \tau^{2}$ ranging from 8 to 40 for different SNR values; $\mathbf{b}$ the average values of $\operatorname{imag}\left(\psi_{3,3}\right)$ versus SNR for different modulations for $u \tau^{2}=40 ; \mathbf{c}$ the average values of real $\left(\psi_{3,3}\right)$ against different $u t^{2}$ ranging from 41 to500for different SNR values; $\mathbf{d}$ the average values of real $\left(\psi_{3,3}\right)$ versus SNR for different kinds of signals for $u \tau^{2}=41$

$0.21 \times 10^{-4}$ in general as shown in Fig. 5b. Finally, we set $t h_{L F M_{1}}=\frac{0.40 \times 10^{-4}+0.21 \times 10^{-4}}{2}=0.31 \times 10^{-4}$ as the optimal threshold for LFM classification. Meanwhile, as in Fig. 5c, the average value of $\operatorname{real}\left(\psi_{3,3}\right)$ for LFM signal class obtains the maximum at $u \tau^{2}=41$ for $\mathrm{SNR}=5 \mathrm{~dB}$ and the maximum is close to $-0.73 \times 10^{-4}$, and for other signal classes, the minimum of the average values of $\operatorname{real}\left(\psi_{3,3}\right)$ is close to $-0.50 \times 10^{-4}$ obtained at $S N R=-5 \mathrm{~dB}$ from Fig. $5 \mathrm{~d}$. Thus the threshold $t h_{L F M \_2}$ can be set to $\frac{-0.73 \times 10^{-4}+-0.50 \times 10^{-4}}{2}=-0.62 \times 10^{-4}$ to guarantee the correct classification of the LFM signals with the timebandwidth product between 41 and 500 .

\section{Results and discussion}

\subsection{Choice of the modulation signal parameters for the Clustering}

The parameters used for the clustering are shown in Table 2. CR, PW and HS stand for code rate, pulse-width and frequency hop size, respectively. Meanwhile, for BPSK, we use 5 bit Barker codes, the 2FSK and 4FSK are encoded by deterministic codes in order to lower the

Table 2 Parameters for the clustering

\begin{tabular}{lllll}
\hline Signal type & CF & CR & PW & HS \\
\hline MP & $800 \mathrm{MHz}$ & & $1 \mu \mathrm{s}$ & \\
BPSK & $800 \mathrm{MHz}$ & $10 \mathrm{MHz} / \mu \mathrm{s}$ & $1 \mu \mathrm{s}$ & \\
2 FSK & $800 \mathrm{MHz}$ & $10 \mathrm{MHz} / \mu \mathrm{s}$ & $1 \mu \mathrm{s}$ & $100 \mathrm{MHz}$ \\
4 FSK & $800 \mathrm{MHz}$ & $10 \mathrm{MHz} / \mu \mathrm{s}$ & $1 \mu \mathrm{s}$ & $100 \mathrm{MHz}$ \\
\hline
\end{tabular}

effect of deficiency of some codes. Codes are defined as [0

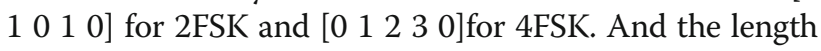
of Rectangular window is set to be 400 . In addition, the SNR values from -5 to $5 \mathrm{~dB}$ for most conditions.

\subsection{Choice of the modulation signal parameters for test}

In order to verify that the proposed method can achieve better performance than the algorithm based STFT, we did the following simulations. The code parameters for BPSK、2FSK and 4FSK are same as the parameters set in 6.1 for both algorithms. The CFs are $800 \mathrm{MHz}$, $1000 \mathrm{MHz}$ and $1600 \mathrm{MHz}$. The HSs are $60 \mathrm{MHz}$, $100 \mathrm{MHz}$ and $1000 \mathrm{MHz}$. The chirp rates for LFM are $40 \mathrm{MHz} / \mu s, 80 \mathrm{MHz} / \mu s, 100 \mathrm{MHz} / \mu s, 1200 \mathrm{MHz} /$ $\mu s$. And the length of rectangular window is set to be 400 and the SNR values from -5 to $5 \mathrm{~dB}$.

\subsection{Simulation results analysis}

To estimate the classifier performance, 50 signals are used for the clustering, and each simulation was run 100 times, evaluating the average recognition rate (ARR).

\subsubsection{The effects of CFs and HSs variation}

Figure 6 is used to get indications how much the CFs and $\mathrm{HSs}$ variation affect the performance at $\mathrm{SNR}=5 \mathrm{~dB}$ and $\mathrm{SNR}=0 \mathrm{~dB}$ respectively. The MP、BPSK and LFM signals are simulated with CFs ranging from $800 \mathrm{MHz}$ to $1600 \mathrm{MHz}$, the FSK signals are simulated with HSs ranging from $60 \mathrm{MHz}$ to $1000 \mathrm{MHz}$. As expected, the variation in CFs and HSs would not affect the performance 

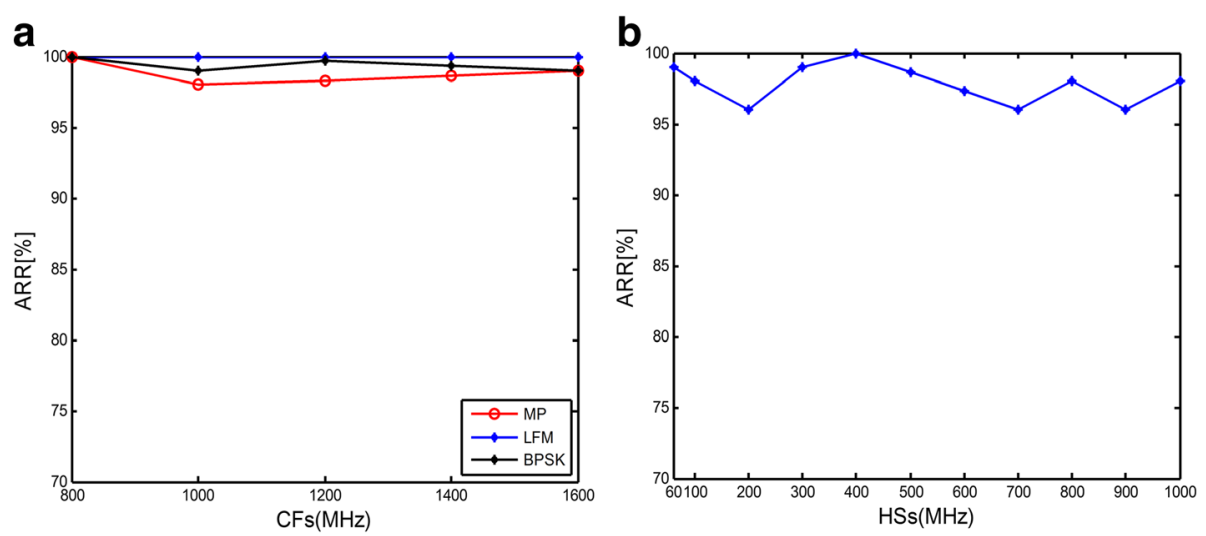

Fig. 6 The effects of CFs and HSs variation: (a) Performance analysis for different CFs for MP、BPSK and LFM signals at SNR =5dB; (b) Performance analysis for different HSs for FSK signals at $S N R=0 d B$

much. And the property makes the pseudo-Zernike moments very suitable to spectrogram features recognization with random variation in the signal parameters: CFs and HSs.

\subsubsection{The performance of the proposed algorithm}

Figure 7 reports the scatter plots related to $\psi_{2,0}$ and $\psi_{5,1}$ for pseudo-Zernike for all the data of the 4 types modulation signals for different SNR. Both the STFT and ST-RFT based algorithms have been considered. The scatter plots shown in Fig. 7a and c demonstrate that for $\mathrm{SNR}=5 \mathrm{~dB}$, the extraction of the pseudo-Zernike moments from STRFT give a certain degree of separation within the class. As shown in Fig. $7 b$ and $d$, for $S N R=-5 d B$ the 4 classes could be considered to be classified more accurate by the proposed algorithms in comparison to the STFT based algorithm. Consequently, in the presence of noise, the
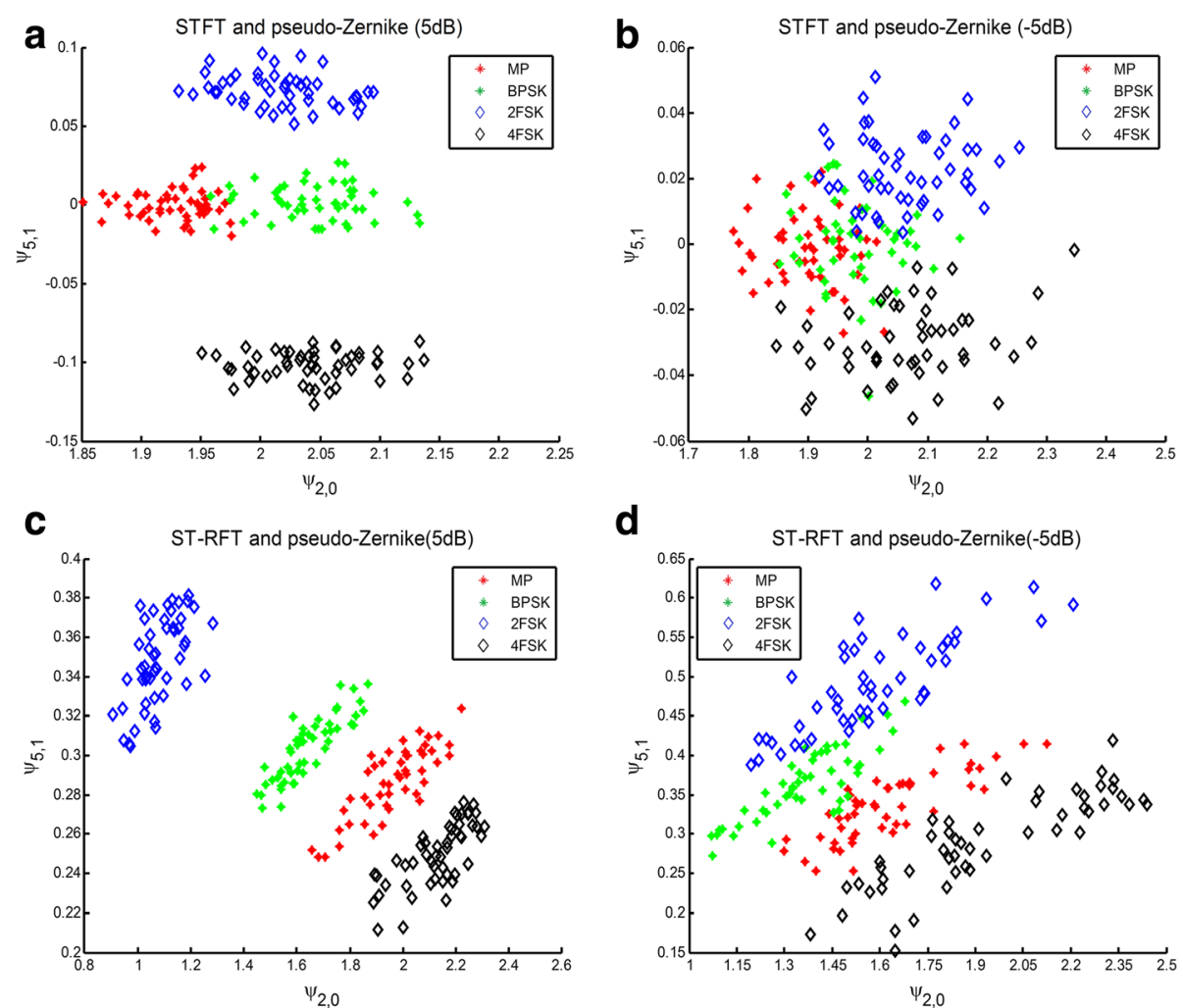

Fig. 7 Scatter plots of 5th vs 27th moments of 4 type modulation signals classes based on ST-RFT and STFT: (a) STFT based algorithm for SNR = $5 \mathrm{~dB}$; (b) STFT based algorithm for SNR = -5 dB; (c) ST-RFT based algorithm for SNR = $5 \mathrm{~dB}$; (d) ST-RFT based algorithm for SNR =-5 dB 
proposed algorithm performs well especially for MP and BPSK signals recognition.

These behaviors are also confirmed by the results illustrated in Fig. 8, where the ARR is plotted versus different SNRs ranging from $-5 \mathrm{~dB}$ to $5 \mathrm{~dB}$ both for STFT, STRFT based algorithms and the algorithm presented in [9]. Thereinto, for STFT and ST-RFT algorithms, the modulations used to obtain the ARR are MP, LFM, BPSK, 2FSK and 4FSK signals satisfying the signal parameters for test discussed in the paper. And for [9], the modulations used to obtain the ARR are the signals of their own choosing. It is obvious that, an increment in the SNR leads to a higher performance. Obviously, in the case of $\mathrm{SNR}=-5 \mathrm{~dB}$, the proposed algorithm reaches a ARR of 90\%, while the ARR of STFT algorithm reaches $70 \%$,and the ST-RFT based algorithms assure a higher level of ARR than the STFT counterpart. Consequently, as conclusion to these analyses, it can be claimed that the performance of our algorithm based on the combination of the ST-RFT and the pseudo-Zernike moments is preferred to STFT based algorithm. Meanwhile, comparison of the work to the techniques presented in [9] shows that the approach proposed in this paper has better robustness against SNR variation.

\section{Conclusions}

In this paper, we have presented a new method for intrapulse modulation recognition under low SNR environment. In this method, the ST-RFT spectrograms for 5 modulation schemes are firstly calculated. Then the pseudo-Zernike moments are applied to the ST-RFT spectrogram to uniquely discriminate the spectrogram features for different modulations when parameters change. Simulation results demonstrate a robust recognition performance over a wide range of SNRs, CFs, HSs, and time-bandwidth product. Meanwhile, based on

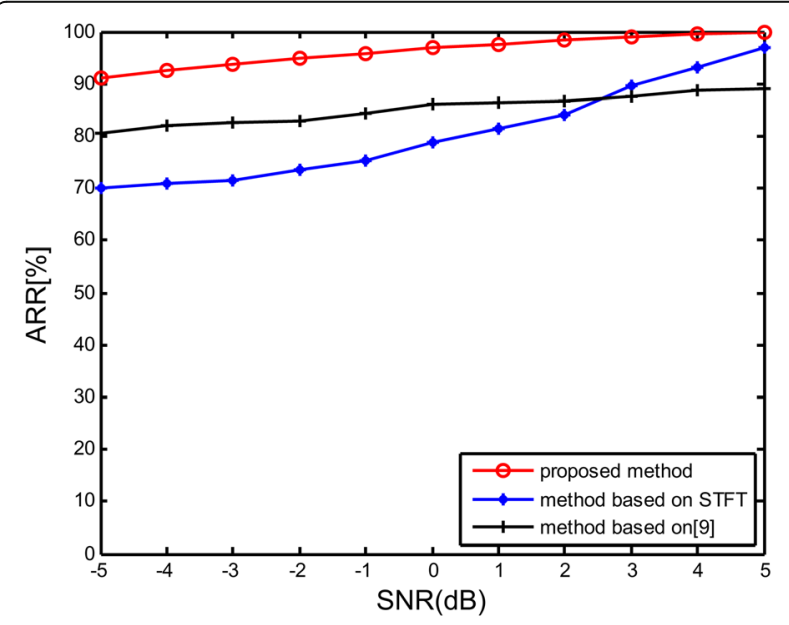

Fig. 8 ARR (\%) versus different SNRs for different algorithms the simulation results analysis, our method is better comparison of the work to the STFT based technique and the technique presented in [9].

However, our work only on few modulation schemes, a discussion on the technique applied to other modulation schemes, such as NLFM, PWM, PPM will be developed.

\section{Abbreviations}

2D: Two-dimensional; 2FSK: Binary frequency shift keying; 4FSK: 4-ary frequency shift keying; ASK: Amplitude shift keying; ARR: Average recognition rate; BPSK: Binary phase shift keying; CF: Carrier frequency; CR: Code rate; DFT: Discrete Fourier Transform; FB: Feature based; FSK: Frequency shift keying; GCD: Greatest common divisor; HS: Hop size; HT: Hough transform: KNN: K-nearest neighbour; LFM: Linear frequency modulation; MP: Monopulse; M-PSK: M-ary phase shift keying; OLR: Overlap rate; PW: Pulse-width; PSK: Phase shift keying; PSR: Probability of successful recognition; QPSK: Quadrature phase shift keying; RS: Ramanujan sums; RFT: Ramanujan Fourier Transform; STFT: Short-time Fourier transform; ST-RFT: Short-Time Ramanujan Fourier Transform; SF: Sampling frequency; SNR: Signal-to-noise ratio; WVD: Wigner-Ville distribution

\section{Acknowledgements}

The authors would like to thank the reviewers for their time and effort spent in carefully reviewing the manuscript, and for their valuable comments that have greatly contributed to the enhancement of article's quality.

\section{Funding}

This work is partially supported by Tianjin Research Program Application Foundation and Advanced Technology (15JCQNJC01100).

\section{Authors' contributions}

XM conceived the approach; XM, DL and YS designed the experiments; DL and YS performed the experiments. All authors read and approved the final manuscript.

\section{Competing interests}

The authors declare that they have no competing interests.

\section{Publisher's Note}

Springer Nature remains neutral with regard to jurisdictional claims in published maps and institutional affiliations.

Received: 3 December 2016 Accepted: 24 April 2017

Published online: 02 June 2017

\section{References}

1. W Pei, QZ Yang, Z Jun, T Bin, Autonomous radar pulse modulation classification using modulation components analysis. EURASIP J. Adv. Signal Process. 2016(1), 1-11(2016)

2. OA Dobre, A Abdi, Y Bar-Ness, W Su, Survey of automatic modulation classification techniques: classical approaches and new trends. IET Com 1(2), 137-156 (2007)

3. D Grimaldi, S Rapuano, LD Vito, An Automatic Digital Modulation Classifier for Measurement on Telecommunication Networks. IEEE Trans Instrum Meas 56(5), 1171-1720 (2007)

4. SZ Hsue, SS Soliman, Automatic modulation classification using zero crossing. IEE Proc, Radar, Sonar Navig 137(6), 459-464 (1990)

5. H Alharbi, S Mobien, S Alshebeili, F Alturki, Automatic modulation classification of digital modulations in presence of HF noise. EURASIP J Adv Signal Process 1, 3639-3654 (2012)

6. K Hassan, I Dayoub, W Hamouda, M Berbineau, Automatic Modulation Recognition Using Wavelet Transform and Neural Networks in Wireless Systems. EURASIP J Adv Signal Process 1, 1-13 (2010)

7. S Qian, D Chen, Joint Time-Frequency Analysis. IEEE Sig Process Mag 16(2), 52-67 (1999)

8. F Hlawatsch, GF Boudreaux-Bartels, Linear and quadratic time-frequency signal representations. IEEE Sig Process Mag 9(2), 21-67 (1992) 
9. Z Yang, W Qiu, H Sun, A Nallanathan, Robust Radar Emitter Recognition Based on the Three-Dimensional Distribution Feature and Transfer Learning. Sensors 16(3), 1-14 (2016)

10. G Lopez-Risueno, J Grajal, A Sanz-Osorio, Digital Channelized Receiver Based on Time-Frequency Analysis for Signal Interception. IEEE Trans Aerosp Electron Syst 41(3), 879-898 (2005)

11. Y Zhang, X Ma, D Cao, Automatic Modulation Recognition Based on Morphological Operations. Circuits Syst Signal Process 32(5), 2517-2515 (2013)

12. D Zeng, H Xiong, J Wang, B Tang, An Approach to Intra-Pulse Modulation Recognition Based on the Ambiguity Function. Circuits Syst Signal Process 29(6), 1103-1122 (2010)

13. D Zeng, X Zeng, H Cheng, B Tang, Automatic modulation classification of radar signals using the Rihaczek distribution and Hough transform. IET Radar Sonar Navig 6(5), 322-331 (2012)

14. TJ Lynn, AZ Sha'amerr, Automatic analysis and classification of digital modulation signals using spectrogram time frequency analysis. Proc. International Symposium on Communications \& Information Technologies (CIT'07) (Sydney, NSW,2007), pp. 916-920.

15. F Xie, C Li, G Wan, An Efficient and Simple Method of MPSK Modulation Classification. 4th International Conf. on Wireless Communications, Networking and Mobile Computing (WiCOM '08) (,Dilian, China, 2008), pp. 1-3

16. M Planat, H Rosu, S Perrine, Ramanujan sums for signal processing of lowfrequency noise. Phys Rev E 66(5), 715-720 (2002)

17. LT Mainardi, L Pattini, S Cerutti, Application of the Ramanujan Fourier Transform for the Analysis of Secondary Structure Content in Amino Acid Sequences. Meth Inf Med 46(2), 126-129 (2007)

18. S Samadi, MO Ahmad, MNS Swamy, Ramanujan Sums and Discrete Fourier Transforms. IEEE Signal Process Lett 12(4), 293-296 (2005)

19. L Sugavaneswaran, S Xie, K Umapathy, S Krishnan, Time-Frequency Analysis via Ramanujan Sums. IEEE Signal Process Lett 19(6), 352-355 (2012)

20. J Lundén, V Koivunen, Automatic Radar Waveform Recognition, IEEE Journal of selected topics in signal process. 1(1), 124-126 (2007)

21. C Clemente, L Pallotta, AD Maio, JJ Soraghanand, A Fatina, A Novel, Algorithm for Radar Classification Based on Doppler Characteristics Exploiting Orthogonal Pseudo-Zernike Polynomials. IEEE Trans Aerosp Electron Syst 51(1), 417-430 (2015)

22. MW Aslam, Z Zhu, AK Nandi, Automatic Modulation Classification Using Combination of Genetic Programming and KNN. IEEE Trans Wireless Commun 11(11), 2742-2750 (2012)

23. S Ramanujan, On certain trigonometric sums and their applications in the theory of numbers. Trans Cambridge Philos Soc 22(13), 259-276 (1918)

24. PP Vaidyanathan, Ramanujan Sums in the Context of Signal Processing -Part I: Fundamentals. IEEE Trans on Signal Process 62(16), 4145-4157 (2014)

25. RD Carmichael, Expansions of arthmetical functions in infinite series. Proc London Math Soc 34(13), 1-26 (1930)

26. M Planat, M Minarovjech, M Saniga, Ramanujan sums analysis of longperiod sequences and 1/f noise. Epl 85(4), 4005-4010 (2008)

27. A Khotanzad, YH Hong, Invariant Image Recognition by Zernike Moments. IEEE Trans Pattern Anal Mach Intell 12(5), 489-497 (1990)

28. R Mukundan, KR Ramakrishnan, Moment Functions in Image Analysis- Theory and Applications (River Edge, NJ, 1998), pp. 1-150

29. K Dae-Won, KH Lee, D Lee, On cluster validity index for estimation of the optimal number of fuzzy clusters. Patt Recog 37(10), 2009-2025 (2004)

30. Z Hong, Q Jiang, H Guan, F Weng, Measuring overlap-rate in hierarchical cluster merging for image segmentation and ship detection. Proc. International Conference on Fuzzy Systems \& Knowledge Discovery(FSKD'07) (,Haikou, Hainan, China, 2007), pp. 420-425

\section{Submit your manuscript to a SpringerOpen ${ }^{\circ}$ journal and benefit from:}

- Convenient online submission

- Rigorous peer review

- Immediate publication on acceptance

- Open access: articles freely available online

- High visibility within the field

- Retaining the copyright to your article

Submit your next manuscript at $\gg$ springeropen.com 